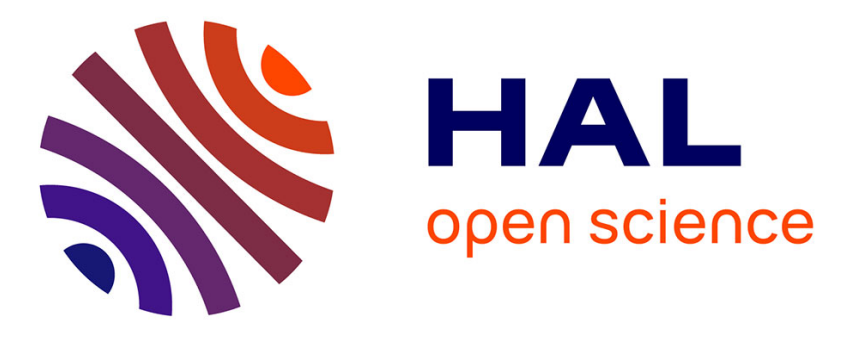

\title{
Transparency-oriented passivity control design for haptic-enabled teleoperation systems with multiple degrees of freedom
}

Gianni Bianchini, Joao Bimbo, Claudio Pacchierotti, Domenico Prattichizzo, Olmo A Moreno Franco

\section{To cite this version:}

Gianni Bianchini, Joao Bimbo, Claudio Pacchierotti, Domenico Prattichizzo, Olmo A Moreno Franco. Transparency-oriented passivity control design for haptic-enabled teleoperation systems with multiple degrees of freedom. CDC 2018 - 57th IEEE Conference on Decision and Control, Dec 2018, Miami Beach, FL, USA, United States. pp.1-6, 10.1109/CDC.2018.8618970 . hal-01875432

\author{
HAL Id: hal-01875432 \\ https://hal.inria.fr/hal-01875432
}

Submitted on 17 Sep 2018

HAL is a multi-disciplinary open access archive for the deposit and dissemination of scientific research documents, whether they are published or not. The documents may come from teaching and research institutions in France or abroad, or from public or private research centers.
L'archive ouverte pluridisciplinaire HAL, est destinée au dépôt et à la diffusion de documents scientifiques de niveau recherche, publiés ou non, émanant des établissements d'enseignement et de recherche français ou étrangers, des laboratoires publics ou privés. 


\title{
Transparency-oriented passivity control design for haptic-enabled teleoperation systems with multiple degrees of freedom
}

\author{
Gianni Bianchini ${ }^{1}$, João Bimbo ${ }^{2}$, Claudio Pacchierotti ${ }^{3}$, Domenico Prattichizzo ${ }^{1,3}$, and Olmo A. Moreno ${ }^{2}$
}

\begin{abstract}
This paper presents a novel control algorithm for haptic-enabled bilateral teleoperation systems involving several degrees of freedom. In particular, the contribution focuses on the implementation of a passivity layer for an established time domain scheme. The proposed approach aims at preserving transparency of interaction along subsets of the environment space which are preponderant for the given task, while guaranteeing the energy bounds required for passivity. The effectiveness of the proposed design is validated via an experiment performed on a virtual teleoperated environment.
\end{abstract}

\section{INTRODUCTION}

The primary goal in the control of haptic-enabled teleoperated robotic systems is to ensure a stable and transparent implementation. Indeed, it is well-known that force feedback can lead to an unstable and therefore unsafe behaviour of the overall system. This can be due to factors such as very rigid contacts, delays in the communication, and a relaxed grasp by the user. These behaviors must be prevented, especially in fields where safety is a paramount and non-negotiable requirement, such as medical robotics [1]. On the other hand, transparency is also important as it represents the match between the impedance perceived by the user and that of the teleoperated environment [2], [3], [4].In this respect, haptic feedback has been shown to play an important role in improving the performance of teleoperation systems in terms of peak and mean force [5], completion time of a given task [6], [7], and accuracy [8]. Therefore, guaranteeing stability while preserving transparency is a prominent control challenge. In this context, passivity theory [9], [10], has been recognized as an effective tool for achieving a stable interaction. In [11], [12], passivity is analysed in the time domain in terms of energy levels of system components. In [13], the problem of making a delayed communication channel passive is addressed. Energy-bounding algorithms to guarantee passivity of the teleoperation loop have been proposed in [14], [15], [16].

In [17], a two-layer control scheme based on the concept of energy tanks is proposed, in which a transparency layer computes the ideal forces to be actuated at both the master and the slave sides; while a passivity layer modulates such forces when this is necessary to avoid violations of the passivity condition, thus guaranteeing stability at the price of a temporary loss of transparency. Along the same line, in [18], the tank-based approach is used to passively reproduce a time-varying stiffness, while in [19] the problem of optimal use of energy in a wave-based teleoperation architecture is addressed.

This work builds upon the two-layer architecture proposed in [17]. In that paper, the design of the passivity layer does

\footnotetext{
${ }^{1}$ Dipartimento di Ingegneria dell'Informazione e Scienze Matematiche, Università degli Studi di Siena, 53100 Siena, Italy \{giannibi, prattichizzo\}ediism.unisi.it

${ }^{2}$ Advanced Robotics Department, Istituto Italiano di Tecnologia, 16163, Genova, Italy \{olmo.moreno, joao.bimbo, domenico.prattichizzo\}eit.it

${ }^{3}$ CNRS, Univ. Rennes, Inria, IRISA, 35042 Rennes Cedex, France claudio.pacchierottieirisa.fr
}

not explicitly account for the amount of transparency that is lost due to the stabilizing control action. This issue is of fundamental importance especially in complex teleoperation tasks that involve multiple degrees of freedom (DoF). Indeed, for a particular configuration of a given task, it may be required that transparency, in terms of fidelity of the rendered force along some subset of the task space, be preserved as much as possible, while other components may be significantly altered in order to preserve passivity without compromising the overall task performance. The aim of this paper is to present a design of the passivity layer which targets at maximizing the level of transparency along subsets of the environment space that are preponderant for the given task at a given time, while preserving the energy bounds that are required to guarantee passivity. When control action is necessary in order to keep the overall system passive, a correction to the ideal forces provided by the transparency layer is computed via the solution of a convex quadratic program, which is characterized by modest computational complexity and is amenable to implementation in real time.

The paper is organized as follows. In Section II some preliminary concepts are recalled. The proposed control design is presented in Section III. Experimental validation of the approach is performed in Section IV. Finally, conclusions are drawn in Section V.

\section{A. Notation}

We denote the continuous time index as $t$ and the discrete time index as $k$. For a continuous-time signal $q(t)$, we denote its sampling $q\left(k T_{s}\right)$ with $q(k)$ without ambiguity, being $T_{s}$ the sampling period. The interval from time $k-1$ to time $k$ is indicated as $\bar{k} . H(\bar{k})$ denotes a quantity $H$ pertaining to or being held constant within $\bar{k}$. For a vector or matrix $v, v^{\prime}$ indicates its transpose. The notation $v \odot w$ is used to denote the component-by-component product of $v$ and $w$. $\|v\|$ is the standard Euclidean norm of $v$.

\section{BACKGROUND}

The present work builds upon the control architecture presented in [17], depicted in Fig. 1 and operating at a sampling time $T_{s}$. Physical interaction occurs at user/haptic device (master) and robot/environment (slave) levels. The generalized positions [velocities] of the end effectors of the master and slave device are denoted by vectors $q_{m}\left[\dot{q}_{m}\right]$ and $q_{s}\left[\dot{q}_{s}\right]$, respectively.

The transparency layer implements a position-force controller (PFC). Its role is to compute the ideal force $\tau_{T L s}$ to be actuated at the slave robot and to convey an ideal haptic feedback force $\tau_{T L m}$ to the master, typically a scaled version of the interaction force $\tau_{e}$ sensed at the slave. A possible implementation of the PFC is as follows:

$$
\begin{aligned}
& \tau_{T L m}(k)=\tau_{e}\left(k T_{s}-T\right) \\
& \tau_{T L s}(k)=-K_{p}\left(q_{m}\left(k T_{s}-T\right)-q_{s}(k)\right)-K_{d} \dot{q}_{s}(k)
\end{aligned}
$$




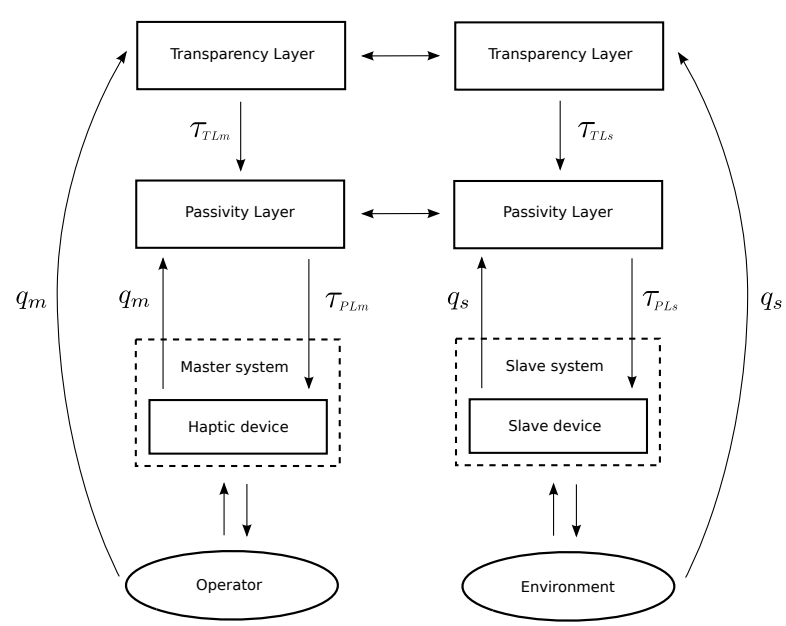

Fig. 1: Controller architecture block diagram.

where $K_{p}$ and $K_{d}$ are suitable proportional and derivative controller gains, and $T$ is the master-slave communication delay (possibly time-varying). The force vectors $\tau_{T L m}$ and $\tau_{T L s}$, once actuated at the respective sides, ensure full transparency to the teleoperation system. The rendering of such forces might not be always achieved in a stable manner depending on the environment and time delays [20].

In [17], passivity of all components of the system is exploited as a viable sufficient condition to ensure overall stability. Indeed, it is well-known that the interaction between passive systems is guaranteed to be stable, and properly combining passive systems results in a passive system [9]. Moreover, the human operator has been shown to preserve stability when interacting with a passive system [21].

For a generic component of a teleoperation system (i.e., the master, the slave, and the communication channel), let $H(t)$ denote its total internal energy. Passivity of such component boils down to the condition that $H(t)$ never exceeds its initial value $H(0)$. If, without loss of generality, zero initial energy is assumed, passivity amounts to the condition

$$
H(t)=\int_{0}^{t} \tau^{\prime}(\sigma) \dot{q}(\sigma) d \sigma \geq 0 \quad \forall t \geq 0,
$$

where $\tau(t)$ and $\dot{q}(t)$ represent the applied external forces and generalized velocities, respectively, or more generally a pair of power-conjugate input/output variables.

Following [17], the total energy $H_{T}(t)$ of the system can be decomposed as

$$
H_{T}(t)=H_{M}(t)+H_{S}(t)+H_{C}(t),
$$

where $H_{M}(t), H_{S}(t)$ and $H_{C}(t)$ denote the energy levels pertaining to the master system, the slave system, and the communication channel, respectively. From (3), passivity of the overall system is therefore achieved if the controller is able to regulate the system for the purpose of ensuring the condition

$$
H_{T}(t) \geq 0 \quad \forall t \geq 0 .
$$

The role of the passivity layer is to suitably modulate the forces $\tau_{T L}$ generated by the PFCs at both sides with the aim of preserving passivity of the overall system, i.e., to ensure that (4) holds at all times. Due to the impossibility of monitoring the overall energy $H_{T}$ in real time owing to the presence of delays, in [17] the following paradigm is adopted. A virtual energy tank $H$ is introduced at each side. Each tank can exchange virtual energy with its counterpart at the other side, and functions as the energy budget available for performing the appropriate (master or slave) force control action. When the tank level at one side is detected as being low, a suitable correction to the ideal actuated force $\tau_{T L}$ is applied by the controller, and the resulting force $\tau_{P L}$ is actuated in order for the tank level not to drop below zero, therefore preserving passivity. This layer also integrates an energy exchange protocol, whose role is to transfer virtual energy packets between the master and slave tanks according to a appropriate balancing algorithm, so as to relax the conservative condition that both tank levels be positive ensuring overall passivity. The details on the latter component are out of the scope of this work and the standard implementation of [17] is used here. Following that, we describe the passivity layer without specific reference to the master or the slave side, as it can be implemented in either, and stick with the notation used in [17].

Let $H(\bar{k})$ represent the available amount of energy to perform the force actuation task during the time interval $\bar{k}$, denote with $q(k)$ be the sampled generalized device displacement, and let $\tau_{r}(\bar{k})$ be the actuated force during $\bar{k}$, which is held constant since a zero-order-hold is used. The energy loss in tank $H$ during $\bar{k}$ is therefore given by

$$
\Delta H(k)=\tau_{r}^{\prime}(\bar{k})(q(k)-q(k-1)) .
$$

Hence, the tank level after the control action has been performed amounts to

$$
H(k)=H(\bar{k})-\Delta H(k) .
$$

Taking into account a possible virtual energy exchange amounting to $H_{ \pm}(k)$, performed according to the exchange protocol, the energy tank level at the end of the time interval $k$ becomes

$$
H(\overline{k+1})=H(k)+H_{ \pm}(k) .
$$

The quantity $H(\overline{k+1})$ in (7) represents the available amount of energy to perform the force actuation task during the time interval $\overline{k+1}$ according to (5).

In the passivity layer implementation proposed in [17], $\tau_{P L}(k)$ is computed by curtailing $\tau_{T L}(k)$ depending on the value of $H(\overline{k+1})$ according to different strategies. However, the problem of suitably shaping $\tau_{P L}$ in the multi-DoF case, depending on the specific task and in order to preserve a certain degree of transparency under the passivity constraint is left as an open question.

\section{PASSIVITY LAYER DESIGN}

In this paper, we propose a design of the passivity layer which addresses the multi-DoF case and aims at preserving transparency as much as possible on suitable subsets of the task space which are relevant to the given task, while guaranteeing passivity.

First, we consider the estimated energy requirement for actuating a perspective control action $\tau_{r}(\overline{k+1})$ as

$$
\Delta \hat{H}(\overline{k+1})=\tau_{r}^{\prime}(\overline{k+1}) \dot{q}(k) T_{s} .
$$

Hence, an estimate of the tank level after the control action has been performed is given by

$$
\hat{H}(k+1)=H(\overline{k+1})-\tau_{r}^{\prime}(\overline{k+1}) \dot{q}(\bar{k}) T_{s} .
$$


Consider a dynamically varying threshold level $H_{\min }(k)>0$, corresponding to the amount of energy to be left in the tank after $\tau_{r}(\overline{k+1})$ has been applied during $\overline{k+1}$, and whose choice is discussed later on. In order for such a level to be guaranteed, according to the estimate in (9), the following constraint must hold:

$$
\hat{H}(k+1)=H(\overline{k+1})-\tau_{r}^{\prime}(\overline{k+1}) \dot{q}(k) T_{s} \geq H_{\min }(k) .
$$

It is easily seen from (10) that if $H(\overline{k+1})-\tau_{T L}^{\prime}(k) \dot{q}(k) \geq$ $H_{\min }(k)$, then the ideal force $\tau_{T L}(k)$ can be safely actuated (i.e., $\tau_{P L}(k)=\tau_{T L}(k)$ ). To address the situation in which this is not possible, $\tau_{P L}(k)$ is computed as the solution of an optimization problem. To this purpose, we denote by $\tau_{C}(k)$ the force correction to be applied by the passivity layer, i.e.,

$$
\tau_{P L}(k)=\tau_{T L}(k)+\tau_{C}(k) .
$$

Let $\mathcal{S}_{i}(k), i=1, \ldots m$ be a suitable set of affine subspaces of the task space, which may depend on the given task, on the current configuration $(q(k), \dot{q}(k))$, and possibly also on the time index $k$. Let us assign a priority index $p_{i}(k) \geq 0$ to each subspace $\mathcal{S}_{i}(k)$, also depending on the current task configuration. The idea of associating each subspace to a priority index is quite simple: the higher the priority $p_{i}(k)$, the stricter the requirement that the projection on $\mathcal{S}_{i}(k)$ of the optimal rendered force $\tau_{P L}(k)$ be as close as possible to the corresponding projection of $\tau_{T L}(k)$. The projection of the force correction $\tau_{C}(k)=\tau_{P L}(k)-\tau_{T L}(k)$ on $\mathcal{S}_{i}(k)$ can be written as

$$
\Pi_{\mathcal{S}_{i}}\left(\tau_{C}(k)\right)=w_{i}(k)+T_{i}(k)\left(\tau_{C}(k)-w_{i}(k)\right),
$$

where the vector $w_{i}(k)$ the projection matrix $T_{i}(k)$ characterize $\mathcal{S}_{i}(k)$. Based on the arguments above, it is natural to define the following functional to be minimized under the tank level control condition (10):

$J\left(\tau_{C}(k)\right)=\sum_{i=1}^{m} p_{i}(k)\left\|w_{i}(k)+T_{i}(k)\left(\tau_{C}(k)-w_{i}(k)\right)\right\|^{2}$.

Note that $J\left(\tau_{C}(k)\right)$ in (12) is quadratic and convex. Furthermore, in most realistic scenarios, it must be ensured that the sign of all components of $\tau_{P L}(k)$ be the same as the corresponding components of $\tau_{T L}(k)$ as a result of the force correction in (11). This constraint prevents the passivity layer from letting the tank gain energy from inverting the direction of rendered forces with respect to $\tau_{P L}(k)$, thus resulting in an excessive loss of transparency. Said another way, the largest allowed correction along each dimension must be to zero out the rendered force. To ensure the latter condition, the following constraint is required:

$\tau_{P L}(k) \odot \tau_{T L}(k)=\left(\tau_{T L}(k)+\tau_{C}(k)\right) \odot \tau_{T L}(k) \geq 0$.

As far as the threshold level $H_{\text {min }}(k)$ is concerned, the following choice is proposed:

$$
H_{\text {min }}(k)=H(\overline{k+1})+\eta\|\dot{q}(k)\|\left(H_{0}-H(\overline{k+1})\right),
$$

where $H_{0}>0$ represents a reference tank level, and $\eta>0$ is a tunable gain parameter. It is easily seen that using $H_{\min }(k)$ in (14) as the threshold in (10) allows the system to recover energy when $H(\overline{k+1})$ is below the reference level $H_{0}$, as well as to bound the spending of energy when it is above. In this respect, it is worth remarking that $H(\overline{k+1})$ is a nondeterministic variable whose value becomes known as soon as $(q(k), \dot{q}(k))$ are sensed and the virtual energy exchange in (7) has taken place. The norm of the current velocity $\|\dot{q}(k)\|$ is also considered in the error gain of (14) in order to limit the correction forces generated by the passivity layer when velocities are low. Motivated by the above observations, we propose the following algorithm as the implementation of the passivity layer. The optimization problem (15) is a convex

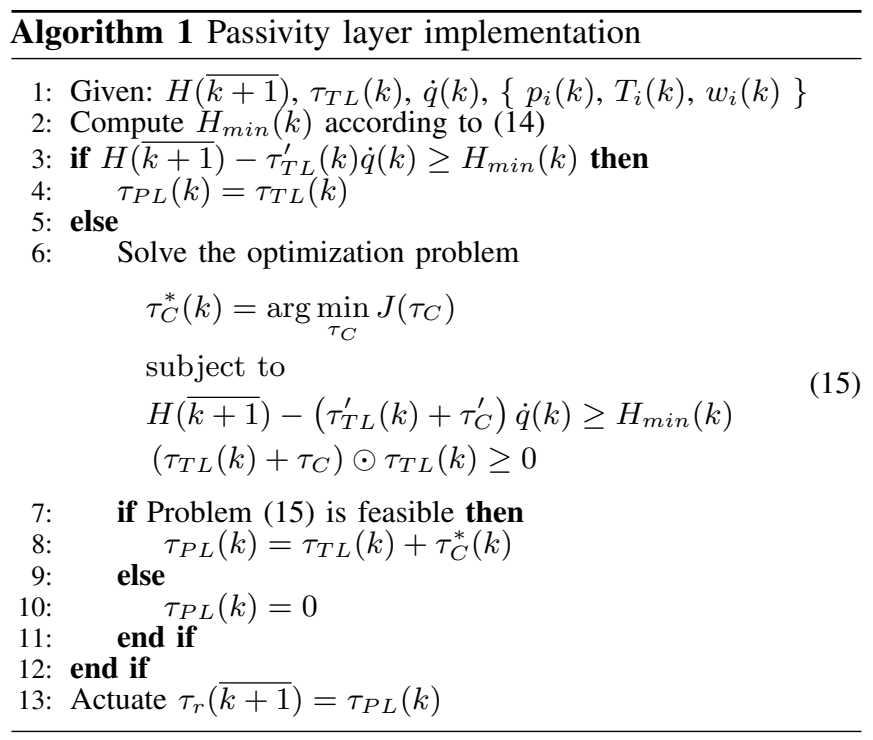

quadratic program. The global minimum can be efficiently computed using interior point methods with a complexity which is quadratic to cubic in the number of variables (i.e., DoFs) involved [22]. On a modern PC, a 6-DoF instance of the problem can be solved in a fraction of a millisecond, thus making the approach compatible with typical sampling frequencies used haptic teleoperation. In the experimental section, the method is implemented without problems on a $3-$ DoF system operating at a sampling frequency of $1 \mathrm{kHz}$.

\section{EXPERIMENTAL RESULTS}

To evaluate the effectiveness of the proposed method, we carried out a palpation experiment. The task consists in palpating a virtual surface and locating a randomly placed circular area which is stiffer than the rest. A comparison of the task performance between the standard implementation of [17] and the proposed one was carried out.

\section{A. Experimental setup}

The experimental setup is depicted in Fig. 2. The master side consists of a grounded 3-DoF Omega haptic device, whose position is linked to the one of the end effector in the virtual teleoperated environment. The control loop operates at a sampling time $T_{s}=1 \mathrm{~ms}$ and is implemented on a GNU/Linux machine using the Robotic Operative System (ROS) framework. The communication channel is modeled as in [17] and simulated by introducing a time delay $T$ (see (1)), which affects the forces and virtual energy exchange between sides. We considered $T$ equal to $5 \mathrm{~ms}$, in addiion to the intrinsic delay of the system, which was measured to be, on average, $1.8 \mathrm{~ms}$. The virtual teleoperated environment is composed of a virtual surface with elastic behaviour along the $z$ axis and displaying a certain amount of friction on the 


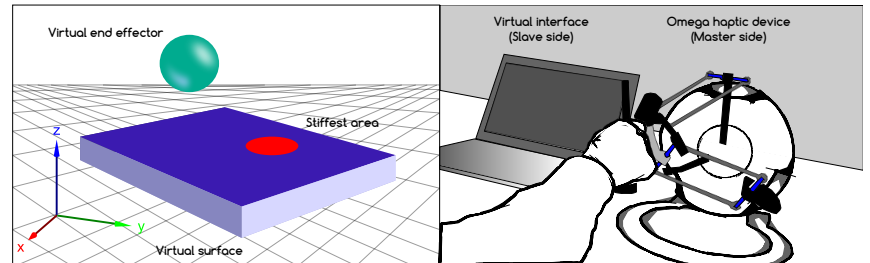

Fig. 2: Experimental setup. On the left, the virtual environment (slave side) shows a contact area (blue surface) with a stiffness value lower than the virtual "bump" (red area). The end effector of the virtual environment is represented by the green sphere, and interacts with the surface, commanded by the master side. On the right side the Omega haptic device is shown.

$x, y$ plane (blue area in Fig. 2). The elastic constant is chosen as $K=1 \mathrm{~N} / \mathrm{cm}$. A stiffer $1-\mathrm{cm}$-radius circular region with elastic constant $K_{H}=2 \mathrm{~N} / \mathrm{cm}$ (red area in Fig. 2) is placed within the surface. When the end-effector enters in contact with the virtual surface, the ideal force rendered on the $z$ axis, normal to the surface, is therefore

$\tau_{e, z}(t)=\left\{\begin{array}{cc}-K_{H}\left(h_{0}-q_{z}(t)\right) & \text { if touching the stiffer area } \\ -K\left(h_{0}-q_{z}(t)\right) & \text { otherwise }\end{array}\right.$

where $h_{0}$ is the $z$ position of the uncompressed surface and $q_{z}(t)$ is the position of the virtual end effector along the $z$ axis. When moving laterally across the surface, along the $x$ and $y$ axes, the user is provided with force feedback $\tau_{e, x}(t)$ and $\tau_{e, y}(t)$, respectively, computed using the standard Coulomb friction model with coefficient $\mu=0.005$. The teleoperation system was tested on the following three modalities and the corresponding performances were compared.

1) No Passivity Controller (NPC): The controller directly transmits the forces and velocities between master and slave and no passivity constraints are enforced. Unmodified forces $\tau_{T L}$ are actuated at both sides.

2) Standard Tank Level Controller (STLC): The controller is implemented as the force curtailing method in [17], where reference values of the relevant parameters are chosen as $\alpha=10$ and $H_{d}=0.2 \mathrm{~J}$.

3) Transparency-Oriented Passivity Layer (TOPL) : The proposed approach described in Sec. III is implemented. Given the nature of the task, i.e., the palpation of the surface to locate a region which is stiffer along the $z$ axis, we find it convenient to define the subspaces $\mathcal{S}_{i}, i=1,2,3$ as the three Cartesian axes $x, y, z$, respectively, and to assign the highest priority to the rendering of the elastic force along the $z$ component, while friction forces on the horizontal $(x, y)$ plane are assigned lower priorities. Reference values of $p_{i}(k)$ are therefore taken to be constant and equal to $p_{1}(k)=p_{2}(k)=0.1, p_{3}(k)=0.5$. Reference values of the parameters in the tank level control policy (14) are chosen as $H_{0}=0.2, \eta=0.1$. For solving the optimization problem (15) in real time, the qpOASES library [23] was used.

For the sake of simplicity, in modalities 2) and 3), the passivity layer was implemented only at the master side. In fact, since the teleoperated environment is virtual, it can be made passive by design. Nevertheless, an energy tank was associated to the slave side anyway. The role of such tank is to be able to provide virtual energy to the master side. In this respect, the energy exchange factor $\beta$ described on Sec. IV-C of [17] is set to 0.01 .

\section{B. Experiment run}

We compare three representative runs of the experiment, one for each modality. The task consisted in moving the end-effector across the virtual surface, passing over the stiffer circular area. The direction of motion therefore evolves along the axes parallel $y$ and perpendicular $z$ to the surface.

The results of the NPC case are depicted in Fig. 3. In the absence of a controller, it is apparent that persistent oscillatory behaviours show up for the considered values of the system parameters.
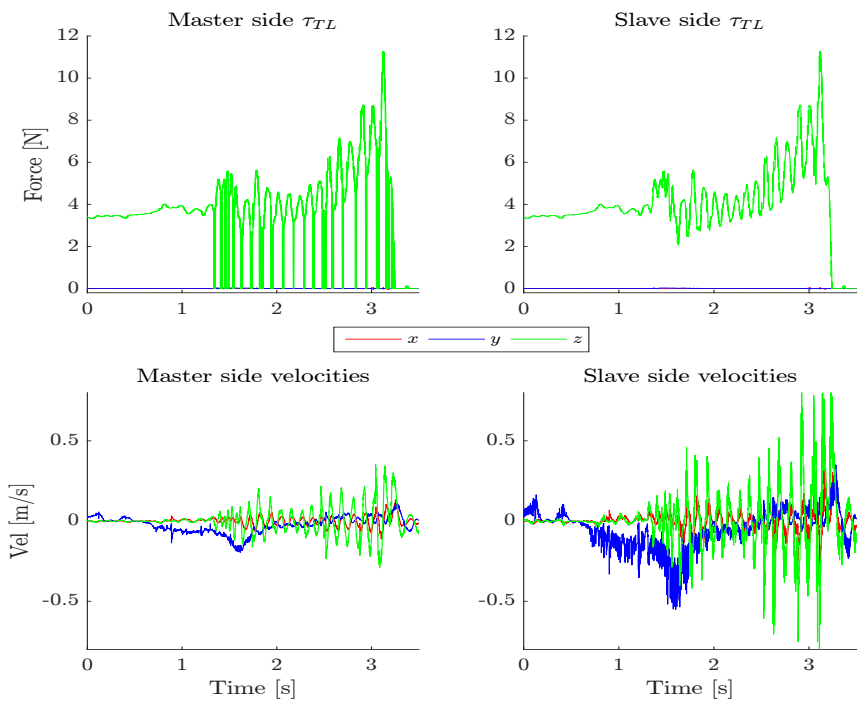

Fig. 3: No Passivity Controller (NPC) case. [Top] Transparency layer forces $\tau_{T L}$ at master (left) and slave side (right). [Bottom] Velocities $\dot{q}$ of the master and slave sides.

The STLC run is represented in Figs. 4 and 5 (the latter figure depicts master-side forces along the $x$ and $y$ axes on a different scale for clarity). The force peak along $z$ corresponds to the end effector passing over the stiffer spot. Note that relevant force corrections are applied by the passivity layer along all axes.

Finally, results from the TOPL run are depicted in Figs. 6 and 7. As opposite to the STLC case, the force correction $\tau_{C}$ introduced by the passivity layer is much more significant along the low-priority directions $x$ and $y$ than along $z$, thus indicating better transparency preservation on the latter subspace.

\section{Perceptual validation}

To validate the efficacy of the proposed control method from a perceptual point of view, we carried out a palpation experiment. The task consisted in palpating the virtual surface to find the center of the stiffer area relying only on haptic cues. The user was asked to grasp the handle firmly. We compared the performance of modalities NPC, STLC and TOPL, each tested for a $T=5 \mathrm{~ms}$ communication delay. To evaluate performance in terms of correct rendering of the environment for the given task, we ran a series of ten repetitions of the experiment for each modality involving a human subject. We registered (i) the error in detecting the center of the stiffer area within the virtual surface, and (ii) the force correction $\tau_{C}$ applied at the master side.

To compare metric (i) between the three modalities, we ran one-way repeated-measures ANalysis of VAriance (ANOVA) 

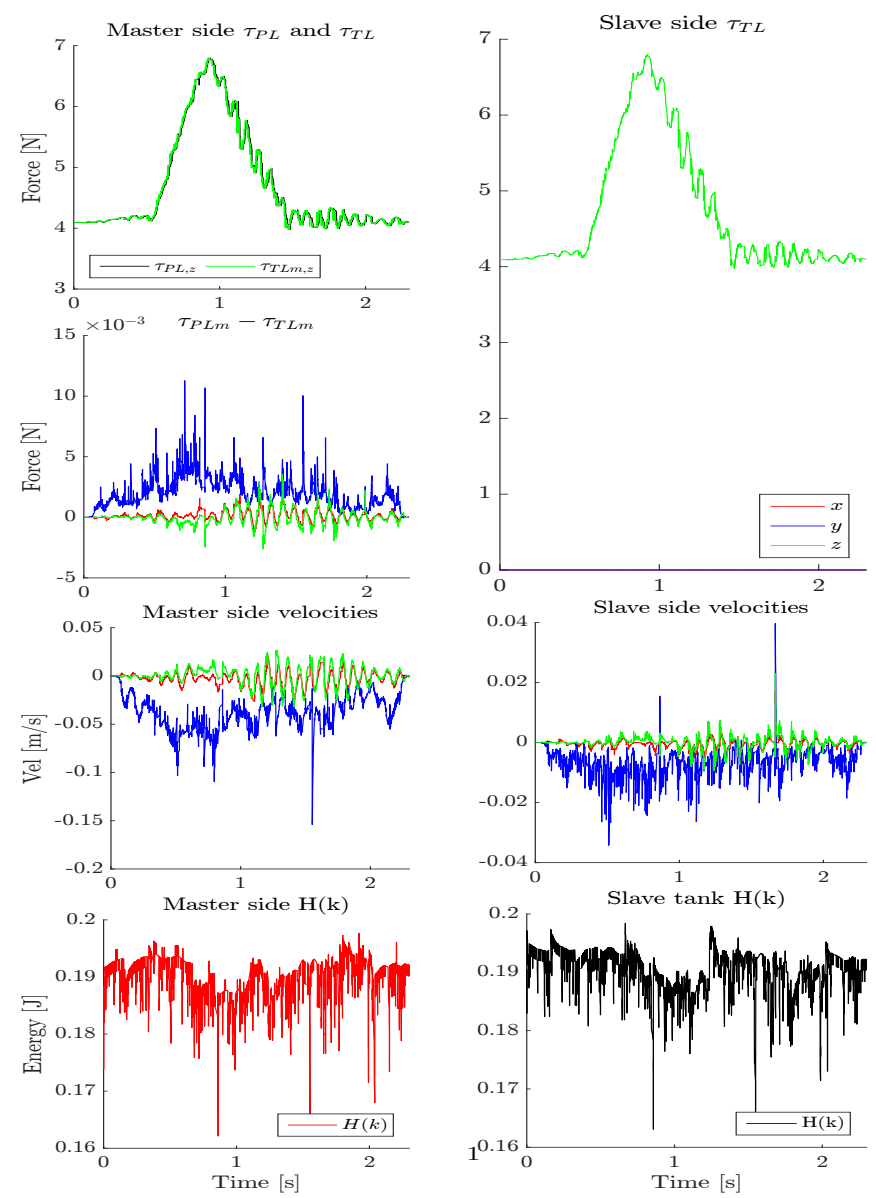

Fig. 4: Standard Tank Level Controller (STLC). [Top] Forces $\tau_{T L}$ and $\tau_{P L}$ at the master (left) and slave (right) side. [Middle-top] Overall force correction $\tau_{P L}-\tau_{T L}$ enforced by the passivity layer at the master. [Middle-bottom] Velocities $\dot{q}$ at both sides. [Bottom] Energy tank levels $H$ at both sides.
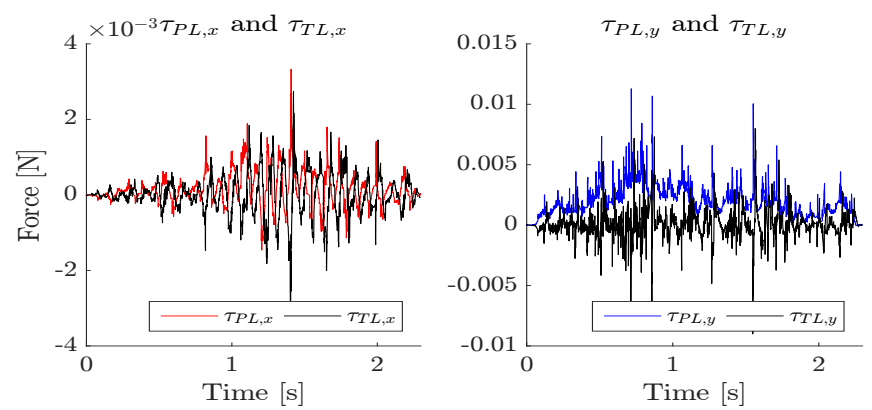

Fig. 5: STLC. Transparency layer $\tau_{T L}$ and passivity layer $\tau_{P L}$ forces at the master side along the $x$ (left) and $y$ (right) axes.

tests on the data. ANOVA is a widely used tool in human subject studies to determine if the mean of two groups can be considered as statistically significant [24]. Fig. 8a shows users' accuracy in terms of detection error when using the three control modalities. All data passed the Shapiro-Wilk normality test, while Mauchly's Test of Sphericity indicated that the assumption of sphericity had been violated for both variables $\left(\chi^{2}(2)=9.337, p=0.009\right)$. In order to account for the non sphericity of our data, we applied a Greenhouse-Geisser correction to the degrees of freedom of ANOVA (see [25] for
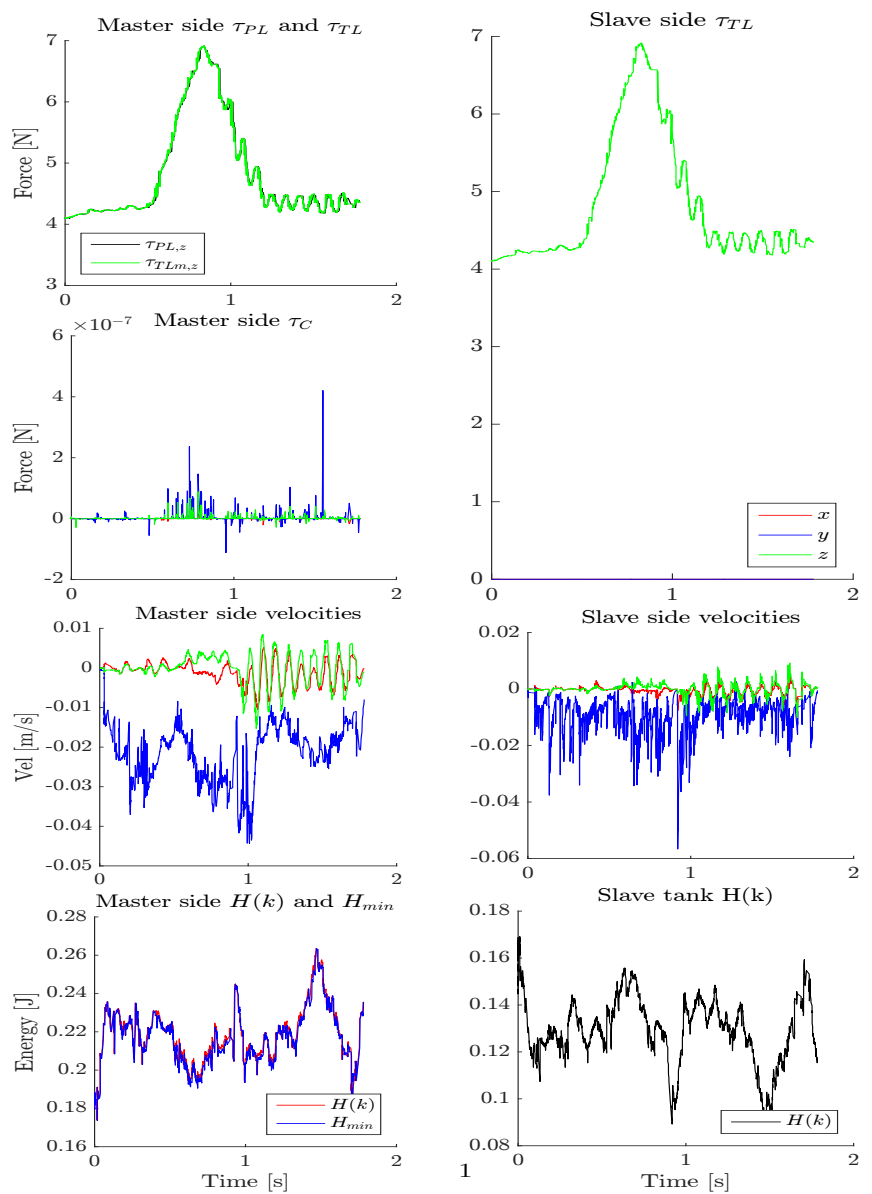

Fig. 6: Transparency-Oriented Passivity Layer (TOPL). [Top] Forces $\tau_{T L}$ and $\tau_{P L}$ at the master (left) and slave (right) side. [Middle-top] Force correction $\tau_{C}$ enforced by the passivity layer at the master. [Middle-bottom] Velocities $\dot{q}$ at both sides. [Bottom-left] Energy tank level $H$ and dynamic threshold value $H_{\min }$ at the master side. [Bottom-right] Energy tank level $H$ at the slave side.
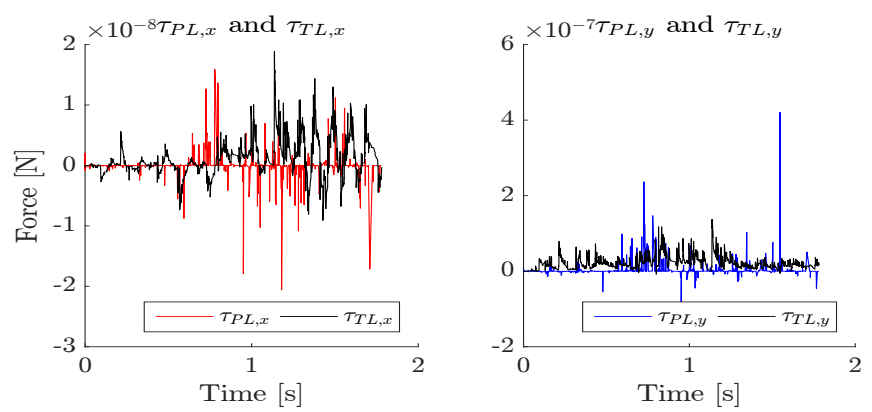

Fig. 7: TOPL. Transparency layer $\tau_{T L}$ and passivity layer $\tau_{P L}$ forces at the master side along the $x$ (left) and $y$ (right) axes.

details on the procedure and on the relevant indices). This test revealed statistically significant difference between the two controllers $\left(F_{1.184,10.659}=17.662, p<0.001, a=0.05\right)$. Post hoc analysis with Bonferroni adjustments revealed a statistically significant difference between NPC and STLC $(p=0.023)$, NPC and TOPL $(p=0.003)$, and between STLC and TOPL $(p=0.004)$. Note that the NPC case with $T=5 \mathrm{~ms}$ time delay gives rise to oscillatory behaviour, 


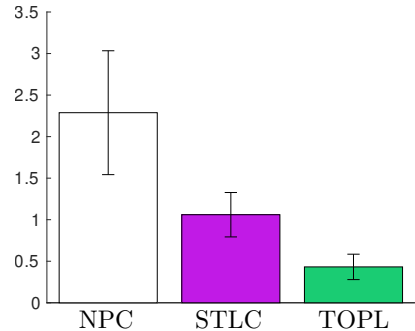

(a) Accuracy (error) in detecting the stiffer area $(\mathrm{cm})$.

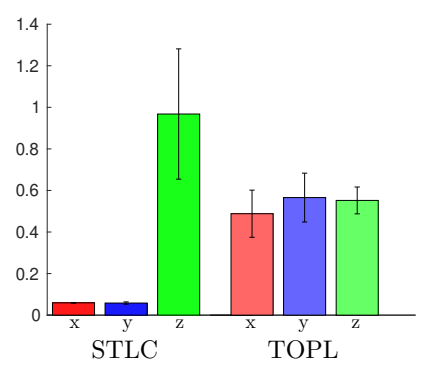

(b) Normalized RMS of the error between ideal and applied forces along the three axes.
Fig. 8: Results of the perceptual validation.

which clearly impacts accuracy. Nevertheless, between the two tested stabilizing controllers, it is apparent that TOPL outperforms STLC.

As far as metric (ii) is concerned, Fig. 8b shows the RMS of the force correction enforced by the controller, i.e., the difference $\tau_{C}$ between the applied $\left(\tau_{P L}\right)$ and ideal $\left(\tau_{T L}\right)$ forces along the three axes, when using the STLC and TOPL control modalities. A two-way ANOVA test can be applied in this case to compare, at the same time, the difference in transparency between the two controllers (STLC vs. TOPL) and among the three axes ( $x$ vs. $y$ vs. $z$ ). All data passed the Shapiro-Wilk normality test. Mauchly's Test of Sphericity indicated that the assumption of sphericity had been violated for the axes variable $\left(\chi^{2}(2)=\right.$ $16.126, p<0.001)$. The two-way repeated-measure ANOVA with a Greenhouse-Geisser correction revealed statistically significant difference in the considered metric among the axes $\left(F_{1.071,9.642}=49.978, p<0.001, a=0.05\right)$ and between the controllers $\left(F_{1,9}=146.559, p<0.001, a=0.05\right)$. Post hoc analysis with Bonferroni adjustments also revealed a statistically significant difference in force correction both between components $\mathrm{x}$ and $\mathrm{z}(p<0.001)$, and between $\mathrm{y}$ and $\mathrm{z}(p<0.001)$. From the latter analysis it is apparent that, whenever transparency reduction is required to reduce transparency to preserve stability, the STLC controller corrects the forces along all axis in a very similar way. Conversely, the TOPL controller corrects less along the privileged $z$ axis, while it significantly corrects the forces along the other axes. This behaviour results in a higher transparency along $z$ for TOPL, thus yielding better task performance.

In addition to the experiment presented here, an extensive set of trials has been conducted for several values of system (time delay) and controller $\left(H_{0}, \eta, H_{d}, \alpha\right)$ parameters. Better performance of TOPL with respect to STLC was obtained in general. Systematic tuning of the parameters involved in the proposed approach is currently being investigated.

\section{CONCLUSION}

A novel passivity control algorithm for haptic-enabled bilateral teleoperation systems with multiple degrees of freedom has been introduced within the framework of [17]. The proposed approach is aimed at enhancing transparency of the interaction along subsets of the environment space designated as the most important ones for the given task, and involves the solution of an optimization problem which is convex and amenable to real-time implementation. The feasibility and effectiveness of the method has been validated on a human subject palpation experiment performed on a virtual environment.

\section{REFERENCES}

[1] J. E. Speich and M. Goldfarb, "Implementation of loop-shaping compensators to increase the transparency bandwidth of a scaled telemanipulation system," in Proc.IEEE International Conference on Robotics and Automation, vol. 3, pp. 2886-2893, 2002.

[2] D. Lawrence, "Stability and transparency in bilateral teleoperation," IEEE Transactions on Robotics and Automation, vol. 9, pp. 624-637, Oct. 1993.

[3] K. Salisbury, D. Brock, T. Massie, N. Swarup, and C. Zilles, "Haptic rendering: Programming touch interaction with virtual objects," in Proc. Symposium on Interactive 3D graphics, pp. 123-130, ACM, 1995.

[4] I. Desai, A. Gupta, and D. Chakraborty, "Transparency enhancement of haptic interface using model matching approach," in Proc. Indian Control Conference, pp. 399-404, Jan. 2016.

[5] C. R. Wagner, S. J. Lederman, and R. D. Howe, "A tactile shape display using RC servomotors," in Proc. Symposium on Haptic Interfaces for Virtual Environment and Teleoperator Systems, pp. 354-355, 2002.

[6] L. Moody, C. Baber, and T. N. Arvanitis, "Objective surgical performance evaluation based on haptic feedback," Studies in Health Technology and Informatics, vol. 85, pp. 304-310, 2002.

[7] C. Pacchierotti, A. Tirmizi, G. Bianchini, and D. Prattichizzo, "Enhancing the Performance of Passive Teleoperation Systems via Cutaneous Feedback," IEEE Transactions on Haptics, vol. 8, pp. 397-409, Oct. 2015.

[8] L. Meli, C. Pacchierotti, and D. Prattichizzo, "Experimental evaluation of magnified haptic feedback for robot-assisted needle insertion and palpation," The International Journal of Medical Robotics and Computer Assisted Surgery, vol. 13, pp. n/a-n/a, Dec. 2017.

[9] R. Lozano, B. Brogliato, O. Egeland, and B. Maschke, Dissipative systems analysis and control. Springer Verlag, 2000.

[10] A. v. d. Schaft, L2 - Gain and Passivity Techniques in Nonlinear Control. Communications and Control Engineering, London: Springer-Verlag, 2 ed., 2000

[11] J.-H. Ryu, D.-S. Kwon, and B. Hannaford, "Stable teleoperation with time-domain passivity control," IEEE Transactions on Robotics and Automation, vol. 20, pp. 365-373, Apr. 2004.

[12] B. Hannaford and J.-H. Ryu, "Time-domain passivity control of haptic interfaces," IEEE Transactions on Robotics and Automation, vol. 18, pp. 1-10, Feb. 2002

[13] G. Niemeyer and J.-J. E. Slotine, "Telemanipulation with Time Delays," The International Journal of Robotics Research, vol. 23, pp. 873-890, Sept. 2004.

[14] J.-P. Kim and J. Ryu, "Robustly Stable Haptic Interaction Control using an Energy-bounding Algorithm," The International Journal of Robotics Research, vol. 29, pp. 666-679, May 2010.

[15] J.-H. Ryu, Y. S. Kim, and B. Hannaford, "Sampled- and continuoustime passivity and stability of virtual environments," IEEE Transactions on Robotics, vol. 20, pp. 772-776, Aug. 2004.

[16] J.-H. Ryu, C. Preusche, B. Hannaford, and G. Hirzinger, "Time domain passivity control with reference energy following," IEEE Transactions on Control Systems Technology, vol. 13, pp. 737-742, Sept. 2005.

[17] M. Franken, S. Stramigioli, S. Misra, C. Secchi, and A. Macchelli, "Bilateral Telemanipulation With Time Delays: A Two-Layer Approach Combining Passivity and Transparency," IEEE Transactions on Robotics, vol. 27, pp. 741-756, Aug. 2011.

[18] F. Ferraguti, C. Secchi, and C. Fantuzzi, "A tank-based approach to impedance control with variable stiffness," in 2013 IEEE International Conference on Robotics and Automation (ICRA), pp. 4948-4953, May 2013.

[19] F. Ferraguti, C. Fantuzzi, and C. Secchi, "Optimizing the use of power in wave based bilateral teleoperation," in 2016 IEEE/RSJ International Conference on Intelligent Robots and Systems (IROS), pp. 1469-1474, Oct. 2016.

[20] M. C. J. Franken, S. Stramigioli, R. Reilink, C. Secchi, and A. Macchelli, "Bridging the gap between passivity and transparency," in Proc. Robotics: Science and Systems, July 2009.

[21] N. Hogan, "Controlling impedance at the man/machine interface," in Proc. International Conference on Robotics and Automation, pp. 16261631 vol.3, May 1989.

[22] V. D. Blondel and J. N. Tsitsiklis, "A survey of computational complexity results in systems and control," Automatica, vol. 36, no. 9, pp. $1249-1274,2000$

[23] H. Ferreau, C. Kirches, A. Potschka, H. Bock, and M. Diehl, "qpOASES: A parametric active-set algorithm for quadratic programming," Mathematical Programming Computation, vol. 6, no. 4, pp. 327363, 2014.

[24] G. R. Iversen and H. Norpoth, Analysis of variance. No. 1, Sage, 1987.

[25] A. Grieve, "Tests of sphericity of normal distributions and the analysis of repeated measures designs," Psychometrika, vol. 49, no. 2, pp. 257267, 1984. 